\title{
Comparison between Optical coherence tomography Angiography and fundus Fluorescein Angiography in cases of Diabetic Maculopathy
}

S.R.Abbas, M.A.Elfayoumi, A.A.Tabl and A.M.M.Zaher

Ophthalmology, Dept., Faculty of Medicine, Benha Univ., Benha, Egypt

E-mail:www.seniorzaher86@gmail.com

\begin{abstract}
Background: Diabetic macular edoema (DME) in industrialised nations has become the most common cause of vision loss. The major cause of serious vision impairment in diabetes individuals is also diabetic macular ischemia (DMI). It is also one of the world's top causes of avoidable blindness, as the WHO states. The objective of this research was to evaluate the state of a capillary bed in diabetic maculopathy in the macular region and the connection between findings with OCT-Angiography and Fundus Fluorescein angiography. Methods: This research consisted of 40 eyes of 25 individuals aged 49 to 70 years with a clinically significant maculo-diabetic edoema as determined by the Early Treatment Diabetic Retinopathy (ETDRS) study without additional eye conditions. Outcomes and conclusion: This research shows the angiographical characteristics of OCT diabetic retinopathy. Some of their benefits over fluorescence angiography include the fast acquirement time, lack of requirement for an intravenous dye, the detection of tiny neovascular tufts and regions of capillary drop-out not masked by leaking. This technique may be helpful for regular diabetic retinopathy monitoring. It has limited field of vision, relatively poor resolution and difficulties identifying micro-aneurysms over a wide region. The lack of the retinal peripheral technology for measuring its vascular health is also a significant barrier to the adoption of OCT-A as the only means of evaluating diabetic retinopathy patients.
\end{abstract}

Keywords: Optical coherence tomography, Angiography, fundus Fluorescein, Diabetic, Maculopathy.

\section{Introduction}

Diabetic retinopathy (DR) is the primary cause of work-age adult visual loss. Blindness rose by $27 \%$ from 1990 to 2010 , and visual impairment by $64 \%$. Diabetic macular edoema (DME) is the most common cause of diabetic visual loss. [1]

Diabetic macular edoema aetiology includes the collapse of the Blood-Retinal Barrier (BRB), including an internal BRB and an external BRB. Diabetic macular edoema is mostly due to the breakup of the internal BRB although there is evidence that external BRB dysfunction may play a role in DME. Breakdown of the BRB provides extravasation into the extracellular space of proteins and other solutes from capillaries. This creates a balance shift in hydrostatic and oncotic pressure, which helps to accumulate fluid in extracellular space and to induce macular edoema. [2]

The key to the clinical diagnosis and grading of the macular edoema is proper clinical examination. Funduscopy with stereopsis and high magnification of all patients with diabetes should be done for the assessment of DME and DR. Another key pillar of diabetic macular edoema diagnosis and monitoring is use of imaging methods that provide a more comprehensive assessment of the disease. These are fluorescein angiography and the tomography of optical coherence (OCT). [3]

Fluorescein angiography is not important for the diagnosis of CLM, but should be done if CSME therapy is contemplated as it differentiates and locates focused regions vs diffuse leakage, guided by this, the use of laser photocoagulation. The closeness of the leak to the foveal avascular zone and the presence or lack of ischemic maculopathy should be observed. [4]

The findings in the macula may be grouped into three patterns: fluorescein fundus angiography (FFA), 1- Focal leakage occurs from microaneurysms and expanded capillary segments and produces focal macular edoema. 2-Late undefined fluorescence leakage occurs from a widespread collapse of the internal blood retinal barrier and produces diffuse macular edoema. The cystoid macular edoema is called 3-Late staining of fovea with pooling the dye into parafoveal cyst, like areas in a petalloid pattern. These patterns may be discovered by themselves or at the same time [5]. Angio-OCT or OCT- angiography is a novel way of analysis based on imagery methods, which allows retinal and choroidal circulation to be visualised without injecting any contrast agent. This novel technique is therefore non-invasive, contrary to the existing gold standard of retinal vascular imagery in Fluorescein angiography. Capillary non-perfusion regions comparable to non-perfused zones shown by fluorescein angiography are visible in individuals with diabetic retinopathy. However, Angio-OCT has high numbers of capillary loops and anastomosis of the arteriovenous. [6]

The objective of this research was to evaluate the state of a capillary bed in diabetic maculopathy in the macular region and the connection between findings with OCT-Angiography and Fundus Fluorescein angiography.

\section{Methods}

This study included 40 eyes of twenty five patients aged from 49 to 70 years with clinically significant diabetic macular edema as defined by Early Treatment Diabetic Retinopathy Study (ETDRS) without any other ocular diseases.

\subsection{Inclusion criteria}

- Age; All patients were from 49 to 70 years.

- Gender; Both male and female were included in the study. 
- Types of diabetes; Type 1 and type 2 diabetes were included in this study.

- Refractive error; Range from $-3 \mathrm{D}$ to $+3 \mathrm{D}$.

- Type of diabetic retinopathy; Mild, Moderate, and severe non-proliferative diabetic retinopathy and proliferative diabetic retinopathy with diabetic maculopathy.

\subsection{Exclusion criteria}

- Patients with previous ocular surgery.

- Patients with previous ocular trauma.

- Patients with previous ocular diseases.

- Patients with history of other vascular diseases e.g: vascular occlusive diseases.

- Patient with previous laser treatment or intravitreal injections.

- Patients with refractive errors beyond the accepted range tailored for the study.

- Increase of intraocular pressure.

2.3. In this prospective randomized study, all patients were subjected to

History taking, including:

Complete ophthalmic evaluation was done including:

Measurement of best corrected visual acuity : using Snellen`s chart, LogMAR acuity testing were obtained from each eye separately ( When using the LogMAR chart, visual acuity is scored with reference to the Logarithm of the Minimum Angle of Resolution, as the chart's name suggests). An observer who can resolve details as small as 1 minute of visual angle scores LogMAR 0, since the base-10 logarithm of 1 is 0 ; an observer who can resolve details as small as 2 minutes of visual angle (i.e., reduced acuity) scores LogMAR 0.3 , since the base-10 logarithm of 2 is nearapproximately 0.3 ; and so on.

Slit limp examination: complete anterior segment evaluation was done.

Measurement of intraocular pressure by goldman applanation tonometer.

Fundus examination: using fundus non-contact lens (VOLK 90D lens).

Fundus photography: The Topcon NW6s NM Fundus Camera.

Fluorescein angiography: Digital retinal camera system (Spectralis HRA2, Heidelberg Engineering, Germany) was used for FA examination after pupillary dilatation with Tropicamide $1 \%$ eye drops to evaluate the perifoveal capillary network, and type of leakage which was categorized into different types:

Focal leakage: Leaking from microaneurysms or capillaries is present on FA.

Diffuse leakage: was diagnosed when poorly demarcated areas of capillary leakage are present on FA.
Cystoid leakage: Diffuse leakage and pooling of dye in the cystic spaces of the macula in the late phase of the angiogram.

Ischemic diabetic maculopathy: the predominant finding in patients with ischemic maculopathy was macular capillary non-perfusion (Enlarged, irregular foveal avascular zone).

Mixed ischemic maculopathy: many patients with ischemic maculopathy have features of more than one type of maculopathy.

Optical Coherence Tomography Angiography (OCT-A): all patients were subjected to (the RTVue XR Avanti, Optovue, Inc., OCT-A)

Diabetic macular edema (DME) also was classified according to retinal morphology detected at accompanied OCT images obtained during OCT-A scans into:

- Focal macular edema.

- Diffuse macular edema.

- Cystoid macular edema.

- Subretinal fluid or neuro-sensory detachment.

Statistical analysis of the data:

Data were analyzed using IBM SPSS software package version 20.0. (Armonk, NY: IBM Corp), Qualitative data were described using numbers and percent. The Kolmogorov-Smirnov test was used to verify the normality of distribution. Quantitative data were described using range (minimum and maximum), mean, standard deviation and median. Significance of the obtained results was judged at the $5 \%$ level. The used tests were Chi-square test For categorical variables, to compare between different groups. Monte Carlo correction; Correction for chi-square when more than $20 \%$ of the cells have expected count less than 5 .

\section{Results}

Our study included 40 eyes of 25 patients. Male to female ratio was 1.2: 0.8 Age ranged between 49 to 70 years old. IDDM to NIDDM ratio was 1.2: 0.8.

In our study, the best corrected visual acuity (LogMar) ranged from 0.20 to 1.30 with mean visual acuity of $0.57 \pm 0.34$. There was significant relation between BCVA (LogMAr) and FAZ diameter with pvalue less than 0.001. Table (2) there was significant relation between BCVA (LogMAr) and foveal density assessed by OCT-A with p value $=0.004$.

In our study, there was significant relation between BCVA (LogMAr) and integrity of FAZ assessed by OCT-A with $\mathrm{p}$ value $<0.001$ Table (4).

In the current study, diabetic retinopathy was classified by fluorescein angiography according to ETDRS into four groups (mild NPDR, moderate NPDR, severe NPDR and PDR). We had 3 eyes with mild NPDR, 7 eyes with moderate NPDR, 16 eyes with severe NPDR and 14 eyes with PDR. Assessment of each group showed in table (5). 
Table (1) Demographic distribution of studied eyes

\begin{tabular}{|c|c|c|}
\hline & No. & $\%$ \\
\hline \multicolumn{3}{|l|}{ Sex } \\
\hline Male & 18 & 45.0 \\
\hline Female & 22 & 55.0 \\
\hline \multicolumn{3}{|l|}{ Age (years) } \\
\hline$<60$ & 16 & 40.0 \\
\hline$\geq 60$ & 24 & 60.0 \\
\hline Min. - Max. & \multirow{3}{*}{\multicolumn{2}{|c|}{$\begin{array}{c}49.0-70.0 \\
58.48 \pm 5.59 \\
58.50\end{array}$}} \\
\hline Mean \pm SD. & & \\
\hline Median & & \\
\hline
\end{tabular}

Table (2) Descriptive analysis of the studied cases according to BCVA (LogMar) $(n=40)$

\begin{tabular}{lccc}
\hline & Min. - Max. & Mean \pm SD. & Median \\
\hline BCVA & $0.20-1.30$ & $0.57 \pm 0.34$ & 0.45 \\
\hline
\end{tabular}

Table (3) Correlation between BCVA and FAZ diameter $(n=40)$

\begin{tabular}{lcc}
\hline & \multicolumn{1}{c}{ BCVA } & \\
& $\mathbf{r}_{\mathrm{s}}$ & $\mathbf{P}$ \\
\hline FAZ Diameter $(\mathbf{m m})$ & $0.604^{*}$ & $<0.001^{*}$ \\
Foveal Density & $-0.451^{*}$ & $0.004^{*}$ \\
\hline
\end{tabular}

\section{$r_{s}$ : Spearman coefficient}

*: Statistically significant at $\mathrm{p} \leq 0.05$

Table (4) Relation between FAZ integrity in OCT-A and BCVA $(n=40)$

\begin{tabular}{lcccc}
\hline BCVA & $\begin{array}{c}\text { Intact } \\
(\mathbf{n = 3 1})\end{array}$ & $\begin{array}{c}\text { FAZ } \\
\text { Ischemic } \\
(\mathbf{n = 9 )}\end{array}$ & $\mathbf{U}$ & $\mathbf{P}$ \\
\hline Min. - Max. & $0.20-1.0$ & $1.0-1.30$ & & \\
Mean \pm SD. & $0.42 \pm 0.21$ & $1.10 \pm 0.15$ & $3.0^{*}$ & $<0.001^{*}$ \\
Median & 0.40 & 1.0 & & \\
\hline
\end{tabular}

\section{U: Mann Whitney test}

$\mathrm{p}$ : $\mathrm{p}$ value for comparing between the different categories

*: Statistically significant at $\mathrm{p} \leq 0.05$

Table (5) Distribution of the studied cases according to fluorescein angiography

\begin{tabular}{lcc}
\hline Fluorescein Angiography & No. & $\%$ \\
\hline FAZ & 31 & 77.5 \\
Intact & 9 & 22.5 \\
Ischemic & & 7.5 \\
Type of DR & 3 & 17.5 \\
Mild NPDR & 7 & 40.0 \\
Moderate NPDR & 16 & 35.0 \\
Severe NPDR & 14 & 47.5 \\
PDR & & 52.5 \\
Macular Edema & 19 & 21 \\
Diffuse & 21 & \\
\hline
\end{tabular}

In this research, 31 eyes out of 40 eyes ( 77.5 percent), without indications of ischemic maculopathy, exhibited macular oedema and 9 eyes out of 40 (22.5 percent) showed a mixed maculopathy for evaluation of macular perfusion. With OCT-A, 25 eyes from 40 eyes $(62,5 \%)$ with intact SCP and DCP without ischemia macula, and 15 eyes from $40(37,5 \%)$ exhibited rarified SCP and DCP without any ischemic macula, and (ischemic macula). In our research, FFA classifies diabetic macular edoema as localised macular edoema and 
diffuse macular edoema. 21 eyes out of $40(52.5 \%)$ exhibited diffuse macular edoema whereas the localised macular edoema was seen in 19 eyes out of $40(47.5 \%)$. Accomplishing OCT pictures from OCT-A scans revealed 7 eyes from $40(17.5 \%)$ had cystoid macular edoema, 15 eyes from $40(37.5 \%)$ had diffuse macular edoema, 15 cases from 40 had macular focal edoema (37.5\%). (7.5 percent ). The centre thickness of macules varied from 206.00 microns to 468.00 microns with a central thickness of $266.4 \pm 50.12$ microns. The foveal capillary density evaluated by OCT-A was $3.30: 41.80 \%$, whereas the average was $14.68 \pm 7.71 \%$ in the present research. FAZ was 0.137 to $0.786 \mathrm{~mm}$ in diameter with a mean FAZ of $0.351 \pm 0.168 \mathrm{~mm}$.

According to the findings, FAZ revealed 31 eyes of 40 (77.5 percent) and 9 eyes of 40 (22.5 percent) with macular ischemia without the indication of ischemia (ischemic maculopathy). While we discovered by OCTA that the macula in the superficial and deep capillary plexuses was ischemic (rariffed FAZ) in 15 eyes out of $40(37.5 \%)$ and this was of statistical significance, since the $\mathrm{P}$ value was less than 0.001 . Macular edoema was divided into two groups, one group, 19 eyes (47.5 percent), and one group, 21 eyes (53.5 percent) had diffuse macular edoema. The FAZ was evaluated individually by OCT-A in each group. The FAZ diameter in the group of eyes with focal macular edoema varied from $0,14 \mathrm{~mm}$ to $0,61 \mathrm{~mm}$, and the FAZ diameter was from $0,22 \mathrm{~mm}$ to $0,79 \mathrm{~mm}$ with the mean FAZ diameter of $0,39 \pm 0,18$. In the second set of eyes, the macular edoema had spread. The FAZ diameter was statistically significantly increased for the diffuse macular edoema eye group in comparison to the other focal macular edoema with a p-value of 0.039. Panel 78

Table (6) Relation between Types of DR by FA and OCT Angiography analysis

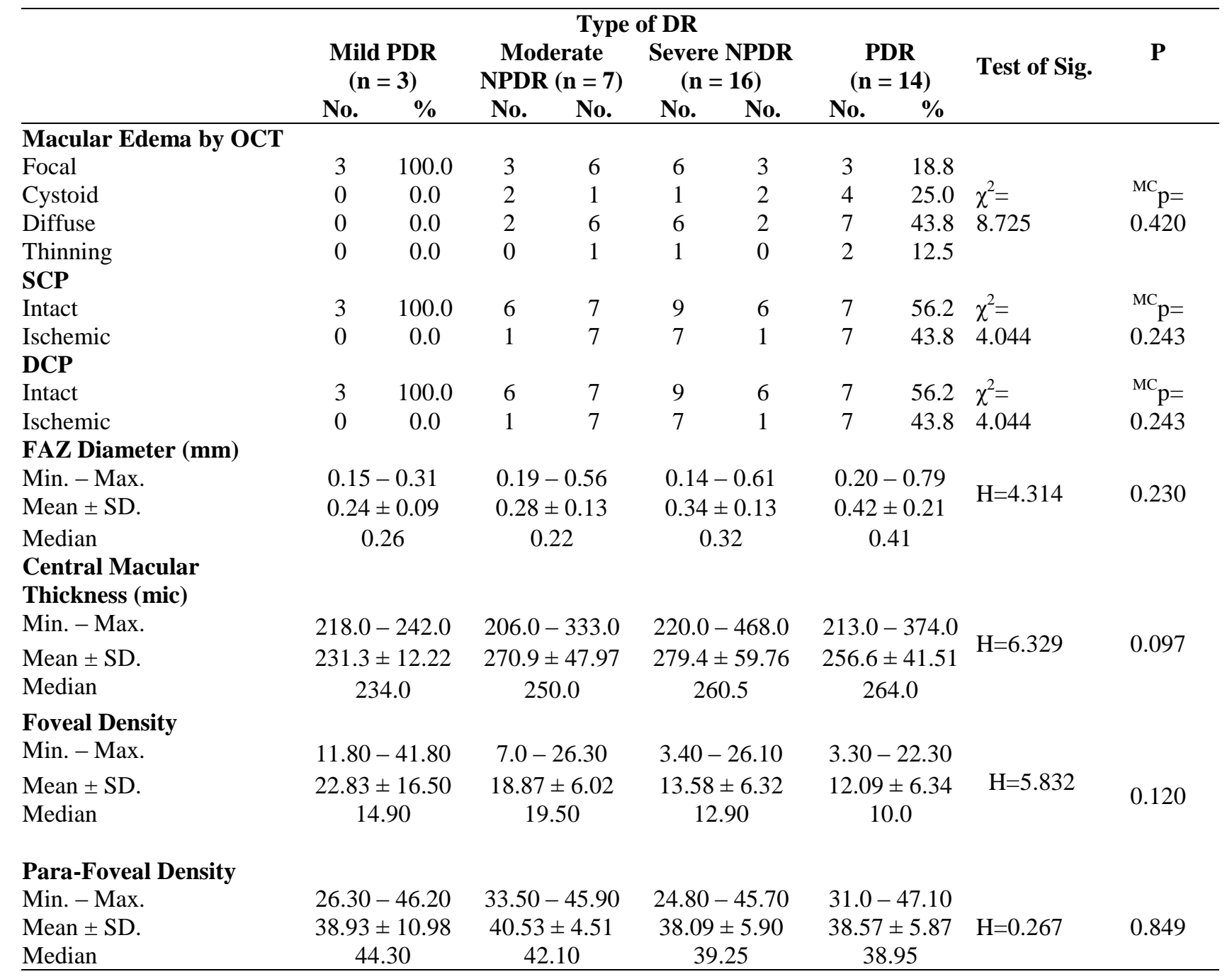


Table (7) Relation between Macular Edema and OCT Angiography ( $\mathrm{n}=40)$

\section{Macular Edema}

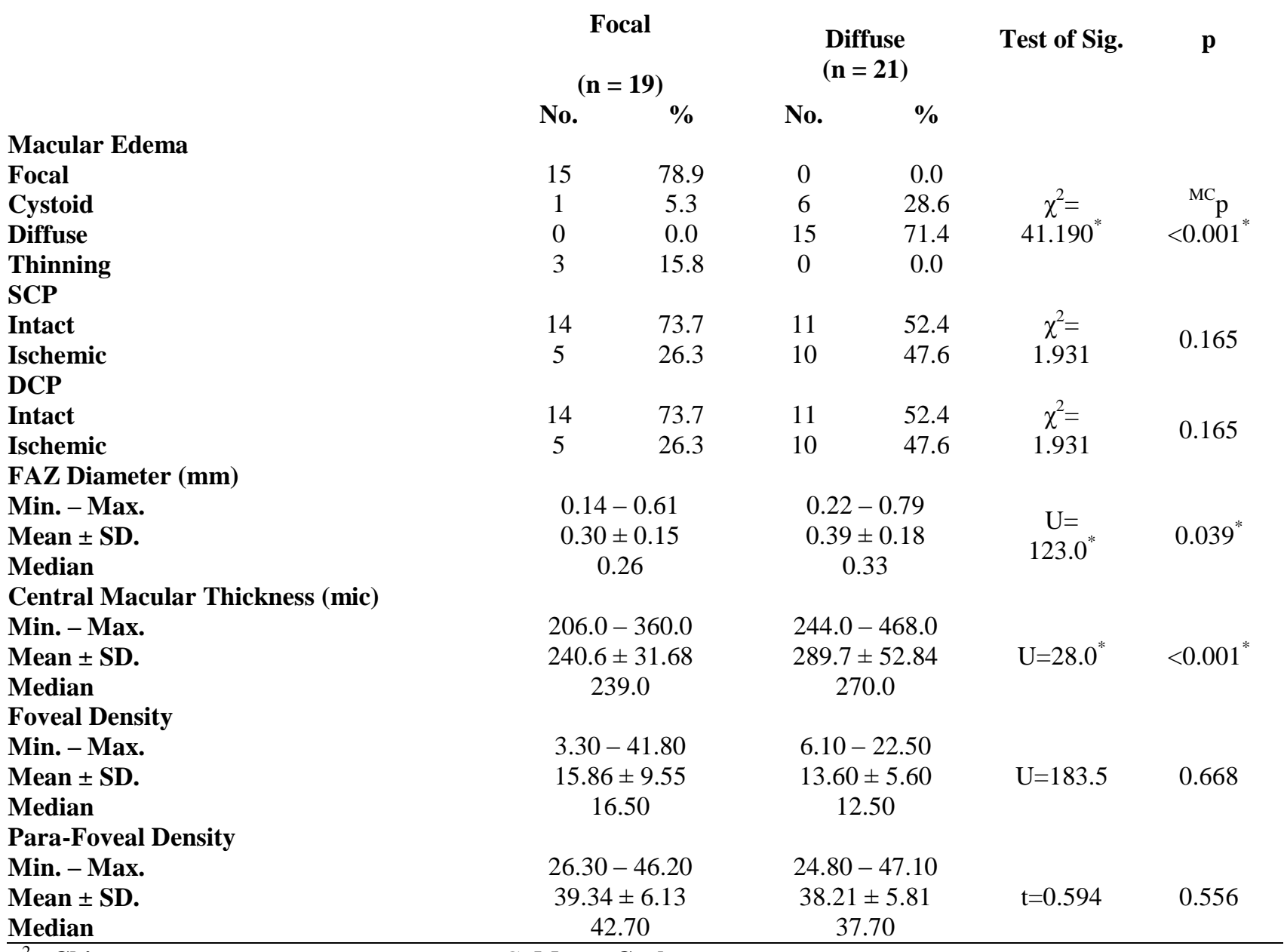

$\chi^{2}$ : Chi square test

t: Student t-test

MC: Monte Carlo

U: Mann Whitney test

$\mathrm{p}$ : $\mathrm{p}$ value for comparing between the different categories

*: Statistically significant at $\mathrm{p} \leq 0.05$

\section{Discussion}

Assessment of fluorescein angiography microvascular alterations has been verified in order to define the severity of the illness and prevent development. Like angiography with fluorescein, an OCT angiography may detect FAZ extension and irregularity and capillary drop regions. [7]

OCT angiography is limited by its narrow field of view. The biggest presently possible view $(6 \times 6 \mathrm{~mm})$ is much less than the normal photography field $(\sim 20$ degrees vs. 30 degrees). Compared to ultra-wide angiography, the change is more striking. Studies have shown that ultra-wide angiography may be more useful in diabetes retinopathy classification, since more disease is seen compared with traditional seven fields of ETDRS. To be sure, a broader field of view would enhance neo-vascularisation sensitivity and show more capillary non-perfusion regions. [8]

OCT-capacity A's to identify diabetic microvascular alterations in comparison to fluorescein angiography was investigated by Couturier et al [9]. They found that OCT-A is superior than FA for the assessment of capillary non-perfusion and may lead to the grading of progression of diabetic maculopathy. This is consistent with the findings in the present research, where OCT-A detects macular ischemia more accurately than FFA.

Jia et al. [10] scanned macular ischemia neovascularization and measured regions of diabetic retinopathy. Their macular ischemia quantification included measurement of foveal avascular area diameter (FAZ) and total non-perfusion area of the artery. This is similar to the findings of our research, which shows a substantial increase in the FAZ diameter in ischemia and non-ischemical maculopathy by OCTA compared to non-isquemic ones.

In non-ischemic patients, the median diameter of FAZ was $0.26 \mathrm{~mm}$ compared to $0.58 \mathrm{~mm}$ for ischemic maculopathy cases. (Satisfactory with a p-value below 0.001). 
Microaneurysms were difficult to detect with the 6 to $6 \mathrm{~mm}$ OCT angiography. Higher resolution but narrower field (3 to $3 \mathrm{~mm}$ ) OCT angiograms may, although not confidently, detect certain microaneurysms. This problem is probably because to the very low micro-aneurysm flow and relatively low scan density utilised in OCT Angiography. Identification of microaneurysms in anguiograms may not be useful in predicting progression risk, but their identification is an integral component of the diabetic macular edoema standard of focused laser treatment [11].

Identification and treatment of all leaky microaneurysms in the 3000 microns in the centre of the macula is part of the usual procedure for the treatment of macular diabetic edoema, which needs at least 6 to $6 \mathrm{~mm}$ of vision. With the introduction of antivascular growth factor therapy and evidence of their superiority to focused laser therapy for edoema centres. Focal laser therapy indications are reduced as the main treatment of diabetic macular edoema. However, it has been determined that FFA can't be substituted by OCT angiography in order to design focused laser photocoagulation [11].

Other vascular properties such as arteriolar wall colour and vascular anomalies showed varied presentations in OCT and FFA. These discrepancies, along with the difficulties of detecting microaneurysms, show the basic difference in how the two methods produce their signal for detection. Contrast in FFA relies on the presence of a colouring, thus the disease that leads to dye buildup, such a micro-aneurysm, is clearer.

Contrast relies on movement or flow in OCT angiography. So, certain angiographical characteristics of fluorescein, such as stain and leak, are not directly equivalent to OCT angiography. However, even in this tiny research, the absence of leakage allowed OCT angiography to identify characteristics that may be hidden by FFA leakage.

More research are necessary to understand the usefulness of these variations in the therapeutic environment of daily life. Although this research has shown that OCT angiography is able to identify many of the characteristics of FFA diabetic retinopathy, it is difficult to draw conclusions about the sensitivity or specificity of the angiography in OCT due to the few patients and the restricted spectrum of diseases.

In order to forecast ranks, choose optimal treatment and monitor the effectiveness of therapy, quantitative evaluation of vascular alterations in diabetic retinopathy is essential. It helps to evaluate the process of vascular remodelling, that may improve our understanding of diabetic retinopathy pathogenesis and assist identify the effectiveness of therapy.

In our research, the foveal vascular density was $16.5 \%$ in non-ischemic patients and $8.3 \%$ in ischemic cases. (Decrease in foveal density statisticianly significant in ischemia patients compared to nonischemic $\mathrm{p}$-value cases $=0.003$ ).

\section{Conclusion}

This research shows the angiographical characteristics of OCT diabetic retinopathy. Some of their benefits over fluorescence angiography include the fast acquirement time, lack of requirement for an intravenous dye, the detection of tiny neovascular tufts and regions of capillary drop-out not masked by leaking. This technique may be helpful for regular diabetic retinopathy monitoring. It has limited field of vision, relatively poor resolution and difficulties identifying micro-aneurysms over a wide region. The fact that the method for the evaluation of its vascular state is not yet applicable to the retinal periphery is therefore a significant barrier to the application of OCT-A as a single measure for the evaluation of diabetes patients.

\section{References}

[1] R.L.Gurung, L.M.FitzGerald, B.J.McComish, N.Verma, and K.P.Burdon, "Identifying genetic risk factors for diabetic macular edema and the response to treatment," J. Diabetes Res., vol. 2020, pp. 1242-1255, 2020.

[2] J. Cunha-Vaz and A.Travassos, "Breakdown of the blood-retinal barriers and cystoid macular edema," Surv. Ophthalmol., vol. 28, pp. 485492, 1984.

[3] D.J.Browning, M.D.McOwen, R.M.Bowen Jr, and L.O.Tisha, "Comparison of the clinical diagnosis of diabetic macular edema with diagnosis by optical coherence tomography," Ophthalmology, vol. 111, pp. 712-715, 2004.

[4] E. T. D. R. S. R. Group, "Early photocoagulation for diabetic retinopathy: ETDRS report number 9," Ophthalmology, vol. 98, pp. 766-785, 1991.

[5] A. Markan, A. Agarwal, A. Arora, K. Bazgain, V. Rana, and V. Gupta, "Novel imaging biomarkers in diabetic retinopathy and diabetic macular edema," Ther. Adv. Ophthalmol., vol. 12, pp. 2515841420950513, 2020.

[6] S. Makita, Y. Hong, M. Yamanari, T. Yatagai, and Y. Yasuno, "Optical coherence angiography,” Opt. Express, vol. 14, pp. 78217840, 2006.

[7] M. B. Peres . "Comparison of optical coherence tomography angiography and fluorescein angiography for the identification of retinal vascular changes in eyes with diabetic macular edema," Ophthalmic Surgery, Lasers Imaging Retin., vol. 47, pp. 1013-1019, 2016.

[8] M. M. Wessel, G. D. Aaker, G. Parlitsis, M. Cho, D. J. D'Amico, and S. Kiss, "Ultra-wide-field angiography improves the detection and classification of diabetic retinopathy," Retina, vol. 32, pp. 785-791, 2012.

[9] A. Couturier ."Capillary plexus anomalies in diabetic retinopathy on optical coherence tomography angiography," Retina, vol. 35, pp. 2384-2391, 2015. 
[10] Y. Jia . "Quantitative optical coherence tomography angiography of vascular abnormalities in the living human eye," Proc. Natl. Acad. Sci., vol. 112, pp. E2395-E2402, 2015.
[11]D. R. C. R. Network, "Elman MJ, Aiello Ll P, Beck RW. Randomized Trial Evaluating Ranibizumab Plus Prompt or Deferred Laser or Triamcinolone Plus Prompt Laser for Diabetic Macular oedema," Ophthalmology, vol. 117, pp. 1064-1107, 2010. 